\title{
Power Balance Optimization Technology of Microgrid Based on Full-Bridge Converter
}

\author{
Shuailong Dai $\mathbb{D}^{1,2}$ Bin Cao, ${ }^{1,3}$ Rui Liu, ${ }^{1,2}$ Liyang $\mathrm{Zhu}^{4}{ }^{4}$ and Mengfan $\mathrm{Li} \mathbb{D}^{1,2}$ \\ ${ }^{1}$ College of Electrical Engineering \& New Energy, China Three Gorges University, Yichang, China \\ ${ }^{2}$ Hubei Province Collaborative Innovation Center for New Energy Microgrid, China Three Gorges University, Yichang, China \\ ${ }^{3}$ Qingyuan Power Supply Bureau of Guangdong Power Grid Inc., Qingyuan, China \\ ${ }^{4}$ Engineering College, Lishui University, Lishui 323000, China \\ Correspondence should be addressed to Mengfan Li; 937644764@qq.com
}

Received 23 May 2019; Accepted 3 September 2019; Published 7 February 2020

Academic Editor: Qingling Wang

Copyright () 2020 Shuailong Dai et al. This is an open access article distributed under the Creative Commons Attribution License, which permits unrestricted use, distribution, and reproduction in any medium, provided the original work is properly cited.

\begin{abstract}
Independent microgrids are widely used in islands and remote townships. However, power imbalance often leads to fluctuations in voltage and frequency, which inhibit the development of AC microgrids. To overcome such problems, this paper proposes an optimized full-bridge converter energy storage structure to realize power balance and optimization of the microgrid. The proposed structure has the characteristics of simple design, easy modularization, and flexible power regulation. First, the working structure and mathematical model are analyzed, and the power model is then established. From the viewpoint of capacitor charging and discharging and inverter, the active and reactive power control technology of the full-bridge structure is analyzed, and a multimode power coordinated control strategy is adopted to adjust and optimize the target power. Finally, the feasibility of the structure and control strategy is verified through a simulation and an experiment. In summary, this study is of great significance to the future promotion and application of AC microgrids.
\end{abstract}

\section{Introduction}

The demand for electricity is growing with the continuous advancement of rural construction [1]. However, in remote mountainous areas and pastoral areas, the environment is harsh, the power load is small and scattered, and in spite of the large investment in the construction of the distribution network, the cycle is long and the loss is large [2-5]. Microgrids constitute a new trend of new energy utilization, featuring wide energy distribution, various forms, and a high utilization rate [4-7]. The use of a microgrid power supply fully exploits various renewable energy sources in rural areas and contributes toward maintaining the stability of the microgrid [8-10]. However, the input of a large number of nonlinear loads and the uncertainty of the wind and light system cause power imbalance in the microgrid [7]. Therefore, the microgrid requires a flexible power compensator to achieve stable operation. A new type of reactive power compensation structure has been proposed $[7,11]$ to realize a substantial continuous reactive output with a small capacitance, but its harmonic content is high and the compensation of active power cannot be realized. Further, a novel double closed-loop control strategy has been proposed $[12,13]$ to inhibit the influence of negative-sequence voltage on the system, improve the switching function, and suppress the third harmonic current, but its control structure is complex and response speed is low. Wang et al. [14] propose a new class of nonlinear PI-based algorithms to relax these requirements and allow for unbalanced and switching topologies having a jointly strongly connected basis. 
Adapting the right nonlinear PI parameters may cause certain difficulties, and its universal applicability deserves further expansion. Ding [15] deals with adaptive consensus output regulation of a class of network-connected nonlinear systems with completely unknown parameters, including the high-frequency gains of the subsystems. In the control design, only the relative information of subsystem outputs is used, provided that regulation error of one of the subsystems is available. Wang and Sun [16] propose a new class of Nussbaumtype function-based algorithms to handle the unknown high-frequency gain signs adaptively and cooperatively in which the underlying topology is a fixed graph with strongly connected. In addition, the energy storage (battery) capacity of the microgrid has been reasonably configured [17] to improve its active power utilization, but the voltage fluctuation caused by the nonlinear load access cannot be resolved. For a clearer comparison, the mainstream reactive power compensators and their characteristics are listed in Table 1.

This paper proposes a full-bridge converter energy storage structure with the characteristics of simple design, easy modularization, flexible power regulation, multimode coordinated operation strategy, and flexible compensation of active power and reactive power of the system. Through parameter optimization, the equivalent power surface of the system is established to meet the requirements of the system's capacitance voltage peak and total current distortion rate, and thus optimize its operation. The circuit structure, working model and principle, and control method of the compensator are described in detail. Finally, a simulation is conducted to prove that the proposed structure can realize flexible power regulation, achieve optimal operation of the system, and guarantee stability of the microgrid.

\section{Full-Bridge Converter Structure and Model}

2.1. Circuit Principle. Figure 1 shows the energy storage structure of a full-bridge converter, which mainly consists of three modules: battery, DC-DC converter, and fullbridge converter. The battery module provides active power and stabilizes the DC side voltage through a DC/ DC converter. The full-bridge converter structure provides flexible power compensation and improves the power quality of the AC side.

2.2. Mathematical Model. As shown in Figure 2(a), the equivalent power model consists of a full-bridge converter voltage source $U_{S} \angle \varphi$ and $A C$ microgrid power source $U_{\mathrm{G}} \angle 0$, where $R$ is the equivalent resistance and $X_{j}$ is the equivalent impedance. As shown in Figure 2(b), the power model of the full-bridge converter is established by vector analysis.

According to the equivalent circuit and vector relationship shown in Figure 2, the full-bridge converter output can be obtained. The incoming current is

$$
\begin{aligned}
& i_{G}=\frac{U_{\mathrm{S}}(\cos \varphi+j \sin \varphi)-U_{\mathrm{G}}}{R+j X}, \\
& S=\dot{U}_{\mathrm{G}} \dot{I}_{\mathrm{G}}=P+j Q, \\
& \left\{\begin{array}{l}
P=\frac{R U_{\mathrm{S}} U_{\mathrm{G}} \cos \varphi-R U_{\mathrm{G}}^{2}}{X^{2}+R^{2}}+\frac{R U_{\mathrm{S}} U_{\mathrm{G}}}{X^{2}+R^{2}} \sin \varphi, \\
Q=\frac{X U_{\mathrm{S}} U_{\mathrm{G}} \cos \varphi-X U_{\mathrm{G}}^{2}}{X^{2}+R^{2}}-\frac{R U_{\mathrm{S}} U_{\mathrm{G}}}{X^{2}+R^{2}} \sin \varphi .
\end{array}\right.
\end{aligned}
$$

As the phase difference between the output voltage phase of the full-bridge converter and the AC grid voltage is small, it can be ignored. Therefore, $\sin \varphi \approx \varphi, \cos \varphi=1$, and the active and reactive power can be expressed as

$$
\left\{\begin{array}{l}
P=\frac{U_{\mathrm{S}} U_{\mathrm{G}}}{X} \varphi, \\
Q=\frac{U_{\mathrm{S}} U_{\mathrm{G}}-U_{\mathrm{G}}^{2}}{X} .
\end{array}\right.
$$

The differential of equation (2) is given by

$$
\begin{aligned}
& \Delta P=\frac{U_{\mathrm{G}}}{X}\left(U_{\mathrm{S}} \Delta \varphi+U_{\mathrm{S}} \varphi+\Delta U_{\mathrm{S}} \Delta \varphi\right), \\
& \Delta \varphi=\int_{0}^{t}\left(\omega_{\mathrm{s}}-\omega_{\mathrm{G}}\right) \mathrm{d} t=\int_{0}^{t} \Delta \omega \mathrm{d} t .
\end{aligned}
$$

Because the value of $U_{\mathrm{S}}$ is much greater than that of $\varphi$, the active power is given by

$$
\Delta P \approx \frac{U_{\mathrm{G}} U_{\mathrm{S}}}{X} \Delta \varphi .
$$

Finally, the active and reactive outputs are

$$
\left\{\begin{array}{l}
\Delta P \approx \frac{U_{\mathrm{G}} U_{\mathrm{S}}}{X} \int_{0}^{t} \Delta \omega \mathrm{d} t \\
\Delta Q \approx \frac{U_{\mathrm{G}}}{X} \Delta U_{\mathrm{s}} .
\end{array}\right.
$$

From equations (4) and (5), it is possible to not only compensate the active power of the AC microgrid to stabilize its frequency but also maintain the stability of the grid voltage by adjusting the reactive power of the system.

\section{Full-Bridge Converter Technology}

3.1. Equivalent Variable Capacitance Technology. Full-bridge converters are mainly used in the grids of cascaded STATCOM modules. However, there are few known applications for microgrids. In view of the power imbalance of microgrids, a new type of equivalent variable capacitor structure is proposed from the perspective of charging and discharging of capacitors. A mathematical model of the equivalent capacitance is established as shown in Figure 3. By controlling the IGBT switch, we can operate it in three different states of charge, discharge, and bypass in one cycle, and thus control the state time of 
TABLE 1: Comparison of mainstream reactive power compensator characteristics.

\begin{tabular}{|c|c|c|c|c|}
\hline \multirow{2}{*}{ Characteristic } & \multicolumn{2}{|c|}{ SVC } & \multirow{2}{*}{ STATCOM } & \multirow{2}{*}{ Full-bridge converter } \\
\hline & TSC & TCR & & \\
\hline Control method & Synchronous & Phase shift & HPWM & Phase shift \\
\hline Control complexity & Easy & Easy & Hard & Easy \\
\hline Modulation frequency & Medium & Medium & High & Medium \\
\hline Switching loss & Medium & Medium & High & Low \\
\hline Responding speed & Medium & Medium & Fast & Medium \\
\hline Compensation accuracy & Low & Medium & High & High \\
\hline Compensation range & Wide & Medium & Wide & Wide \\
\hline Compensation type & Discrete capacitive reactive & Continuous sensible reactive & Continuous reactive & Continuous capacitive \\
\hline Current quality & High & Low & Medium & Medium \\
\hline
\end{tabular}

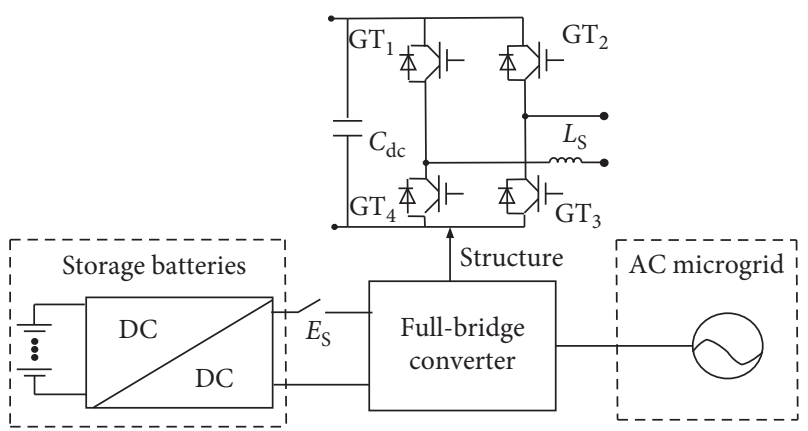

Figure 1: Full-bridge converter energy storage structure.

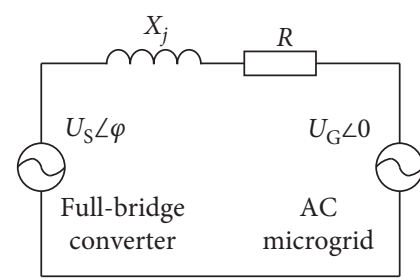

(a)

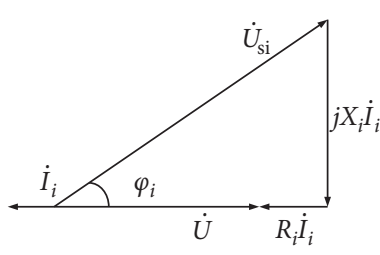

(b)
FIgURE 2: Equivalent power model. (a) Equivalent full-bridge transformation model. (b) Voltage vector analysis.

action to realize an equivalent capacitance with variable capacitance characteristics. The range of variable capacitors is $[0, \infty]$.

Figure 4 shows the working principle diagram of a fullbridge converter. It can be seen that the full-bridge converter operates in three states: charging, discharging, and bypassing. The current and voltage of the full-bridge converter are periodic odd functions.

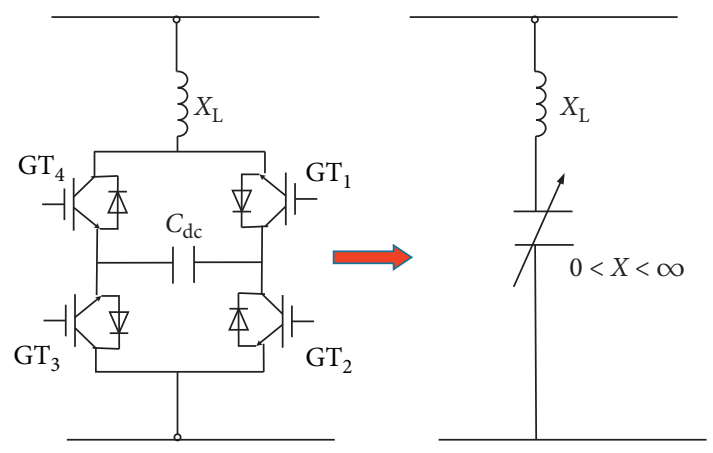

FIGURE 3: Full-bridge equivalent structure.

As shown in Figure 4, by setting the minimum capacitance hysteresis voltage $V_{c-m i n}$, the control tube is turned off in advance to make it enter the single-tube conduction mode, and the charge and discharge state is controlled. Finally, the voltage of the full-bridge converter can be obtained as equation (9), where $X_{C}$ is the capacitance impedance, $I$ is the fundamental current, and $k$ is an integer:

$$
\begin{cases}\sqrt{2} X_{\mathrm{C}} I(\sin x-\sin \alpha)+V_{\mathrm{c}-\min }, & x \in(2 k \pi+\alpha, 2 k \pi+\pi-\alpha), \\ 0, & x \in(k \pi-\alpha, k \pi+\alpha), \\ \sqrt{2} X_{\mathrm{C}} I(\sin x+\sin \alpha)-V_{\mathrm{c}-\min }, & x \in(2 k \pi+\pi+\alpha, 2 k \pi+2 \pi-\alpha) .\end{cases}
$$

Fourier transformation of equation (6) yields the fundamental voltage of the full-bridge converter as equation (7). The equivalent variable impedance is given by equation (8), and the reactive power output from the full-bridge converter is given by equation (9):

$$
\begin{aligned}
& V_{\mathrm{H} 1}=I X_{\mathrm{C}}\left(1-\frac{2 \alpha}{\pi}-\frac{\sin (2 \alpha)}{\pi}\right)+\frac{4 V_{\mathrm{c}-\min } \cos (\alpha)}{\sqrt{2} \pi}, \\
& X_{\mathrm{H}}=X_{\mathrm{C}}\left(1-\frac{2 \alpha}{\pi}-\frac{\sin (2 \alpha)}{\pi}\right)+\frac{V_{\mathrm{c}-\min }}{I} \cdot \frac{4 \cos (\alpha)}{\sqrt{2} \pi},
\end{aligned}
$$




\begin{tabular}{|c|c|c|c|c|c|}
\hline GT1 & GT2 & GT3 & GT4 & Condition & Flow path \\
\hline Off & Off & Off & On & $\begin{array}{l}\text { Forward } \\
\text { charging }\end{array}$ & $\mathrm{e}$ \\
\hline Off & Off & Off & On & $\begin{array}{c}\text { Single-tube } \\
\text { forward bypass }\end{array}$ & $\mathrm{f}$ \\
\hline On & Off & On & Off & $\begin{array}{c}\text { Forward } \\
\text { discharging }\end{array}$ & $\mathrm{a}$ \\
\hline Off & On & Off & On & $\begin{array}{c}\text { Back } \\
\text { discharging }\end{array}$ & b \\
\hline Off & Off & On & Off & $\begin{array}{l}\text { Single-tube } \\
\text { reverse bypass }\end{array}$ & c \\
\hline Off & On & Off & On & $\begin{array}{l}\text { Reverse } \\
\text { charging }\end{array}$ & $\mathrm{d}$ \\
\hline
\end{tabular}

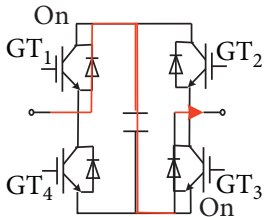

(a) Forward discharging

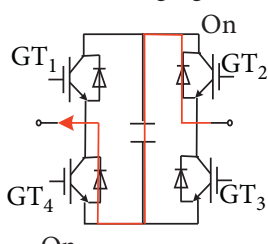

On

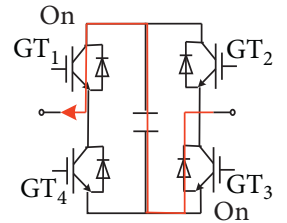

(b) Back

discharging

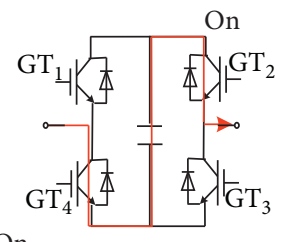

(e) Forward charging

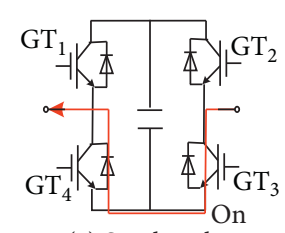

(c) Single-tube reverse bypass

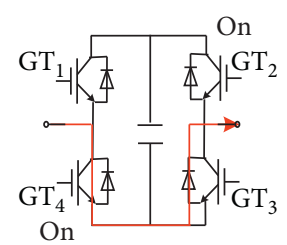

(f) Single-tube forward bypass

FIGURE 4: Full-bridge STATCOM working principle.

$$
Q_{\mathrm{out}}=\frac{U_{\mathrm{m}}^{2}}{X_{\mathrm{H}}-X_{\mathrm{L}}}=\frac{\sqrt{2} U_{\mathrm{m}}^{2} I \pi \omega C}{\left(\sqrt{2} \pi I-2 \sqrt{2} \pi \alpha I-\sqrt{2} I \sin 2 \alpha+V_{\mathrm{c}-\min } 4 \cos \alpha-\sqrt{2} \pi \omega L I\right)}
$$

From equation (9), the greater the values of $\alpha$ and $V_{c-\text { min }}$, the smaller the system and the greater the reactive power output by the system. Conversely, the smaller the values of $\alpha$ and $V_{\mathrm{c} \text {-min }}$, the larger the system and the smaller the reactive power output by the system.

3.2. Full-Bridge Converter Inverter Technology. To ensure power balance of the microgrid, the system needs to compensate not only the reactive power but also the active power. To this end, the voltage outer loop and the power double loop strategy of the inner loop are used to compensate the active power. Figure 5 shows the dual closedloop control technology.

Figure 5(a) is a block diagram of active power closedloop control, where

$$
\left\{\begin{array}{l}
G \operatorname{ppi}(s)=\frac{k_{\mathrm{pp}}+k_{\mathrm{ip}}}{s}, \\
G_{\mathrm{pd}}(s)=\frac{1}{T_{s} s+1}, \\
H_{i}(s)=\frac{U_{0}^{2}}{s L+R} .
\end{array}\right.
$$

In the formula, $G_{\mathrm{ppi}}(s)$ is a transfer function of active power for the PI controller; $H_{i}(s)$ is an equivalent circuit for active power output; $G_{\mathrm{pd}}(s)$ is the transfer function for the sampling delay link; $k_{\mathrm{pp}}$ is the proportional coefficient of PI control on active power; $k_{\text {ip }}$ is the integral coefficient of PI control on active power; and $I_{\mathrm{sc}}$ means the total current flowing into the energy storage module on the DC side. Finally, its active power closed-loop control transfer function $W_{\mathrm{p}}(s)$ is shown in the following equation:

$$
W_{\mathrm{P}}(s)=\frac{U_{0}^{2}\left(k_{\mathrm{pp}} s+k_{\mathrm{ip}}\right)}{s(s L+R)\left(T_{s} s+1\right)+U_{0}^{2}\left(k_{\mathrm{pp}} s+k_{\mathrm{ip}}\right)} .
$$

Figure 5(b) is a block diagram of the outer loop control based on feedforward voltage. The main purpose of the DC capacitor voltage outer loop control is to stabilize the DC side voltage. The parameters in Figure 5(b) is as follows:

$$
\left\{\begin{array}{l}
G_{\mathrm{upi}}(s)=\frac{k_{\mathrm{pu}}+k_{\mathrm{iu}}}{s}, \\
G_{\mathrm{ud}}(s)=\frac{1}{T_{\mathrm{s}} s+1}, \\
W_{i}(s)=\frac{1}{0.5 T_{\mathrm{s}} s+1}, \\
G_{\mathrm{f}}(s)=\frac{\sqrt{2} V_{\mathrm{bn}}}{U_{\mathrm{dc}}} \\
H_{\mathrm{v}}(s)=\frac{1}{s C_{\mathrm{dc}}},
\end{array}\right.
$$

where $G_{\text {upi }}(s)$ is a transfer function of DC voltage for the PI controller; $G_{\mathrm{ud}}(s)$ is the transfer function of the DC voltage for the sampling delay link; $H_{\mathrm{v}}(s)$ is an equivalent circuit for DC voltage; $W_{i}(s)$ is the transfer function of the PWM regulator 


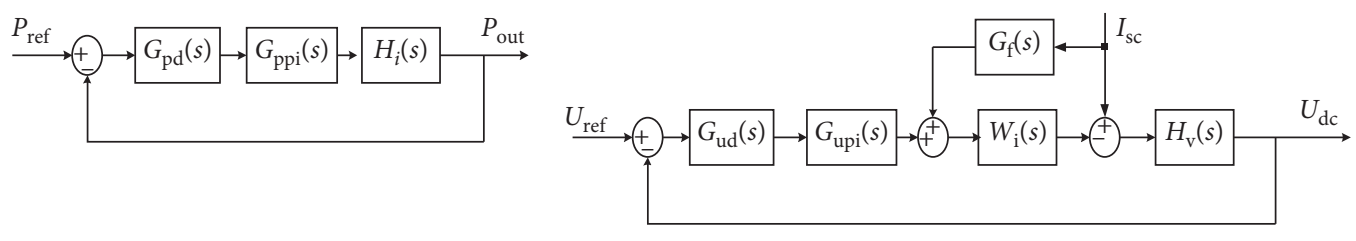

(a)

(b)

Figure 5: Double closed-loop control technology. (a) Power inner loop. (b) Voltage outer loop.

link; $G_{\mathrm{f}}(s)$ is the transfer function of DC/DC side current feedforward control; $k_{\mathrm{pu}}$ is the proportional coefficient of PI control on vpltage; $k_{\mathrm{iu}}$ is the integral coefficient of PI control on voltage; $V_{\mathrm{bn}}$ is the output voltage on the battery side; and $U_{\mathrm{dc}}$ is the voltage of compensator DC side. The transfer function of the DC side voltage outer loop is as follows:

$$
\begin{aligned}
U_{\mathrm{dc}}(s)= & \frac{1 / s C_{\mathrm{dc}} G_{\text {upi }}(s) G_{\mathrm{ud}}(s) W_{i}(s)}{1+1 / s C_{\mathrm{dc}} G_{\text {upi }}(s) G_{\mathrm{ud}}(s) W_{i}(s)} U_{\text {ref }}(s) \\
& +\frac{1-G_{\mathrm{f}}(s) W_{i}(s)}{s C_{\mathrm{dc}}+G_{\mathrm{upi}}(s) G_{\mathrm{ud}}(s) W_{i}(s)} I_{\mathrm{sc}}(s) .
\end{aligned}
$$

When designing its control parameters, the robustness of DC voltage outer loop control should be strengthened. Therefore, when analyzing the voltage loop, it can be considered that the power feedforward completely eliminates the influence of the DC/DC side fast response system on the DC voltage when the active power is required and the bandwidth of the voltage loop is much smaller than the bandwidth of the current inner loop. Therefore, equation (14) can be simplified to

$$
W_{\mathrm{U}}(s)=\frac{k_{\mathrm{pu}} s+k_{\mathrm{iu}}}{C_{\mathrm{dc}} s^{2}+k_{\mathrm{pu}} s+k_{\mathrm{iu}}},
$$

where $k_{\mathrm{pu}}$ is the scale factor for PI, $k_{i}$ is the integral coefficient for PI, and $C_{\mathrm{dc}}$ is a DC capacitor.

\section{Multimode Power Coordination Control}

To achieve coordinated power operation, a multimode power coordinated control strategy is adopted as shown in Figure 6. By sampling the grid voltage, branch current, battery capacity, etc., the actual active and reactive power on the AC microgrid side is calculated and compared with the target active and reactive power. The active and reactive power is realized through the switch controller ES.

When the reactive power is constant, the active power required by the load is insufficient, i.e., $P_{\text {out }}<P_{\text {ref }}$ and $Q_{\text {out }}=$ $Q_{\text {ref }}$. Here, Es is closed, the full-bridge converter is inverted, and the battery is regulated by DC-DC. The output voltage is compared with the given voltage through double closed-loop control to form an instantaneous error adjustment signal. After PI adjustment, feedback is provided to the power inner loop, which then passes through the PI regulator to form a control signal that generates a pulse signal to control the output of the active power.

When the active power of the microgrid is stable and there is a large amount of reactive power in the system, $P_{\text {out }}=P_{\text {ref }}$ and $Q_{\text {out }}<Q_{\text {ref }}$. At this time, Es is disconnected, the phase voltage of the grid is detected by the phase-locked loop, the capacitor voltage is detected at the same time, and the hysteresis capacitor voltage reference value $V_{c-\min }$ is passed. The phase shift angle $\alpha$ controls the $\mathrm{GT}_{1}, \mathrm{GT}_{3}$ and $\mathrm{GT}_{4}, \mathrm{GT}_{2}$ tube turn-on half cycle as well as the $\mathrm{GT}_{1}, \mathrm{GT}_{3}$ tube lag synchronization, while the phase angle $\pi+\alpha$ controls the $\mathrm{GT}_{4}, \mathrm{GT}_{2}$ lag synchronization in a voltage wave period. The phase interval is $[0.5 \pi, 1.5 \pi]$ and the capacitor voltage $V_{\mathrm{c}}$ is less than $V_{\mathrm{c}-\min }$. When the $\mathrm{GT}_{2}$ tube is turned off in advance, only the $\mathrm{GT}_{4}$ tube works; it enters the single-tube bypass state and bypasses the capacitor. Similarly, in the phase interval $[0.5 \pi, 1.5 \pi]$, the $\mathrm{GT}_{1}$ tube is turned off and only the $\mathrm{GT}_{3}$ tube is operated; it enters the single-tube bypass state. The entire reactive control can be changed with $\alpha$. The value of $V_{c-\text { min }}$ compensates for the reactive power of the system and optimizes the system by adjusting the parameters.

When the active power and reactive power of the microgrid do not meet the requirements of the microgrid at the same time, $P_{\text {out }}<P_{\text {ref }}$ and $Q_{\text {out }}<Q_{\text {ref }}$. According to the principle of frequency modulation and voltage regulation, the system is first compensated for active power, $E_{\mathrm{s}}$ is closed, and the compensation is completed. Then, Es is disconnected and reactive power compensation is performed to ensure power balance of the AC microgrid.

Two different working modes are controlled by the power regulator to realize power compensation of active and reactive power. Firstly, the voltage and current data on the busbar are collected to calculate the active and reactive power. When the actual active power value is less than the reference value, the active power is compensated according to the adjustment principle, the switch ES is closed, and the power inner loop is closed. The double closed-loop of the voltage outer loop compensates for the active power; when the active power is sufficient, the ES switch is turned off, the $\alpha$ and $V_{\mathrm{C} \text {-min }}$ are adjusted to achieve the reactive power compensation, and the optimization is adjusted to meet the smaller capacitor voltage and total harmonics of the MERS. The requirement for a lower distortion rate is shown in Figure 7. Finally, the double-closed active compensation and parameter optimization control can be coordinated to ensure the power balance of the bus.

\section{Simulation and Experiment}

5.1. Simulation Analysis. To verify the flexible power adjustment of the microgrid by the full-bridge converter, a simulation model is established. As can be seen from Figure 6 , the structure can be operated in multiple power modes, which can compensate the active and reactive power 


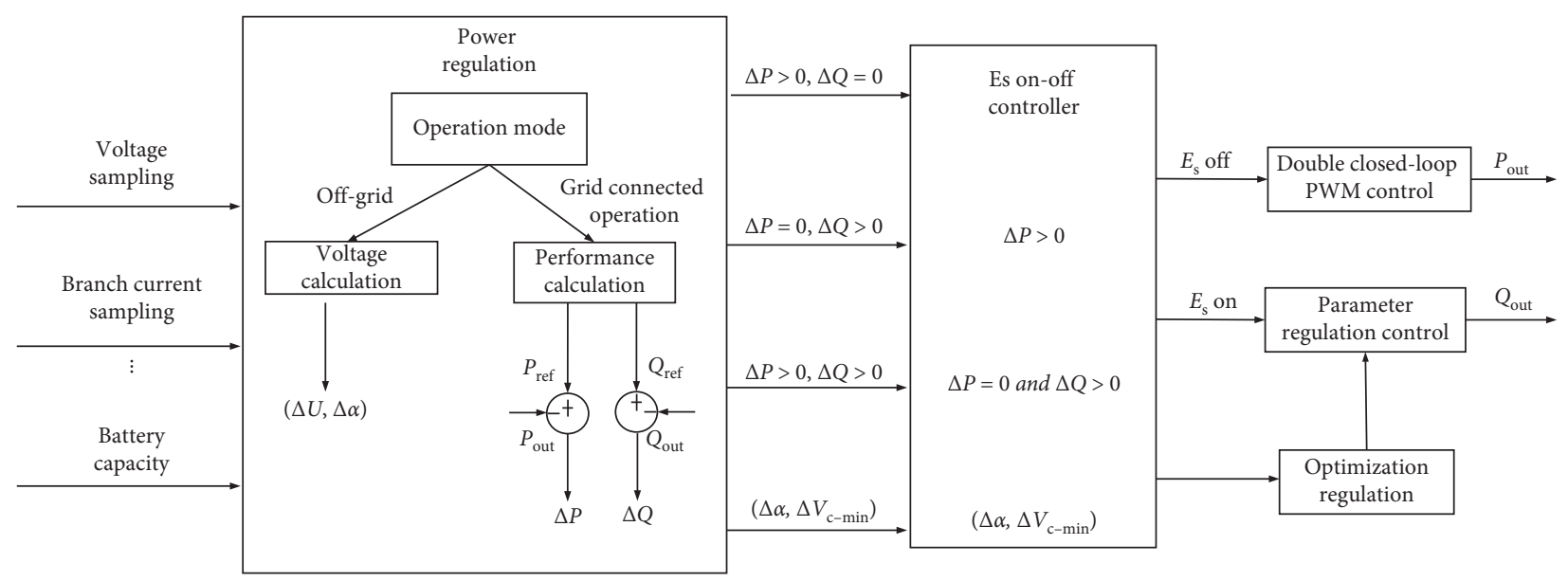

FIgURE 6: Multimode power regulation control.

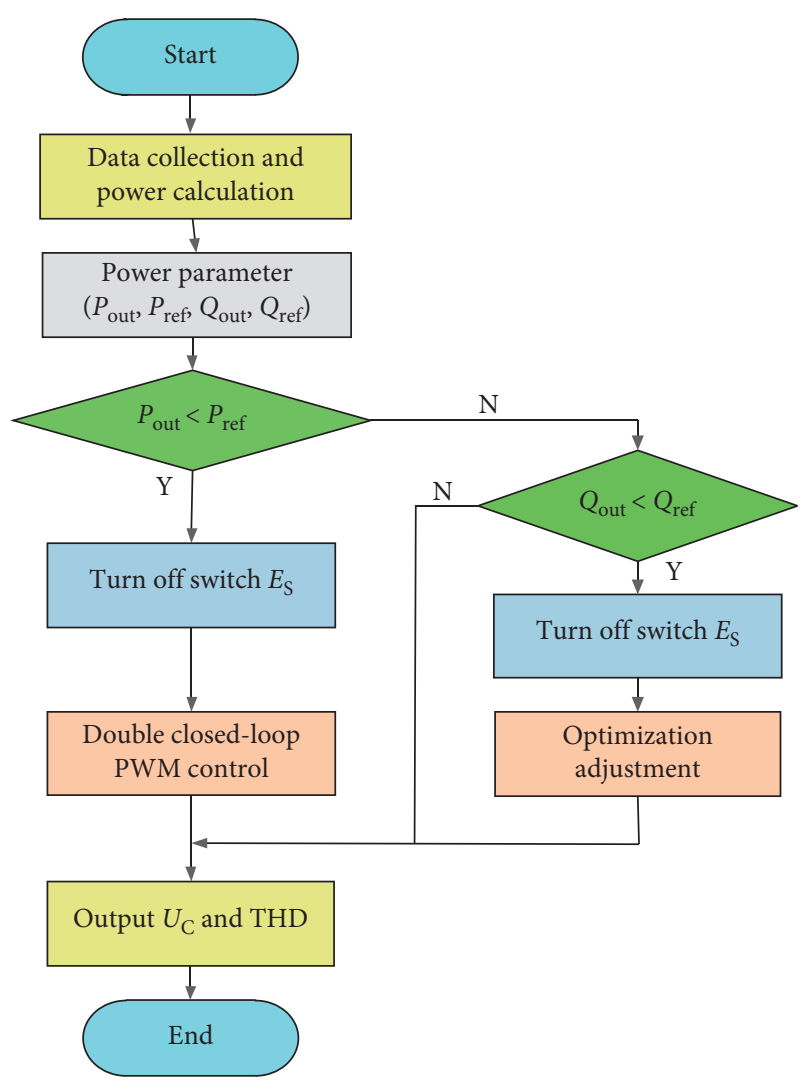

FIGURE 7: A flow chart of proposed control.

of the system. According to the principle of frequency modulation and voltage regulation, first, $P_{\text {out }}<P_{\text {ref }}$ and $Q_{\text {out }}<Q_{\text {ref }}$. For example, the target active power is $6.5 \mathrm{~kW}$ and the reactive power is $2.4 \mathrm{kVAr}$. As shown in Figure $8(\mathrm{a})$, the active power compensation is performed first, and at $0.3 \mathrm{~s}$, the active power of the target output is reached. At this time, the switch Es is turned off, reactive power compensation is performed, and at $0.53 \mathrm{~s}$, the target reactive power is reached. As shown in Figure 8(b), the frequency is gradually stabilized at $50 \mathrm{~Hz}$ from the power frequency of $50.7 \mathrm{~Hz}$. At $0.3 \mathrm{~s}$, owing to the action of the switch Es, the frequency drops sharply and then recovers quickly, and reactive power compensation is performed. At $0.53 \mathrm{~s}$, the voltage frequency is stable. Thus, the simulation verifies that the structure has flexible power adjustment capability to effectively suppress voltage and frequency fluctuations.

To ensure optimal operation of the system and meet the system requirements, this study aims to optimize the microgrid system structure and ensure stability of the system by targeting its capacitor voltage peak and total current distortion rate. To this end, in the simulation experiment, $\alpha$ and $V_{c-\min }$ are the independent variables, and the waveforms of current distortion rate and capacitance voltage value are obtained, respectively, as shown in Figure 9.

Figure 9 plots the total current distortion rate and capacitance voltage peak against the parameters $\alpha$ and $V_{c-m i n}$. As shown in Figure 9(a), the total distortion rate of the current without the third harmonic is lower than that with the third harmonic, and the current distortion rate increases with $V_{c-\min }$ and $\alpha$; however, between $20^{\circ}$ and $60^{\circ}$, the current distortion rate is somewhat reduced. As shown in Figure 9(b), the voltage value of the capacitor increases and decreases linearly with $V_{c-\min }$; when $\alpha$ is greater than $40^{\circ}$, the capacitor voltage will increase exponentially. Through simulation analysis of the parameters, the equivalent reactive power is output for different $\alpha$ and $V_{\mathrm{c}-\min }$. As shown in Figure 10, there is a target reactive point where the peak value of the capacitor voltage is low and the harmonic content is lower. As shown in Figure 11, the optimal target reactive power is $5 \mathrm{kVAr}$.

As shown in Figure 11, equal power curve points $A\left(16^{\circ}, 95 \mathrm{~V}\right)$ and $B\left(27^{\circ}, 180 \mathrm{~V}\right)$ can be obtained by optimizing the comparison before and after. When the target reactive power is the same, the peak value of the capacitor at the optimized point $B$ is lower than the peak voltage at point $A$ before optimization by around $15 \%$. Meanwhile, the harmonic content also shows a certain decrease compared with point $A$. The simulation indicates that adjustment of $\alpha$ and $V_{c-m i n}$ can effectively reduce the capacitor voltage and harmonics, achieve small capacitance of the system, and realize operation with low harmonics. 


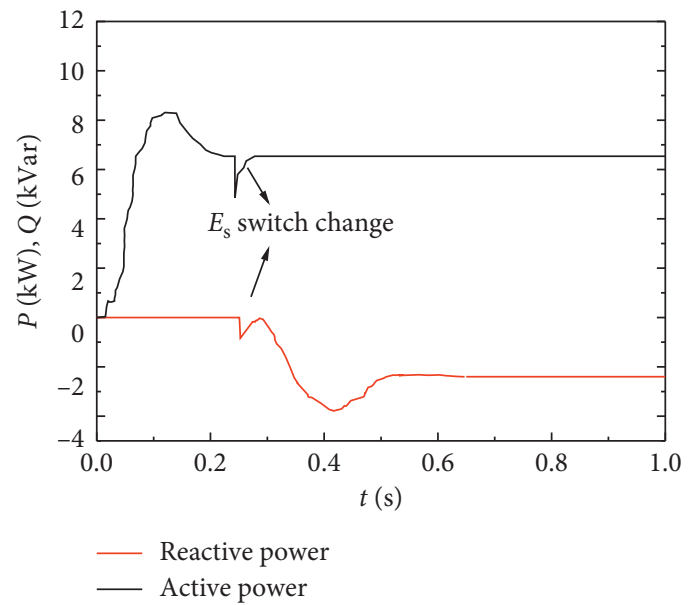

(a)

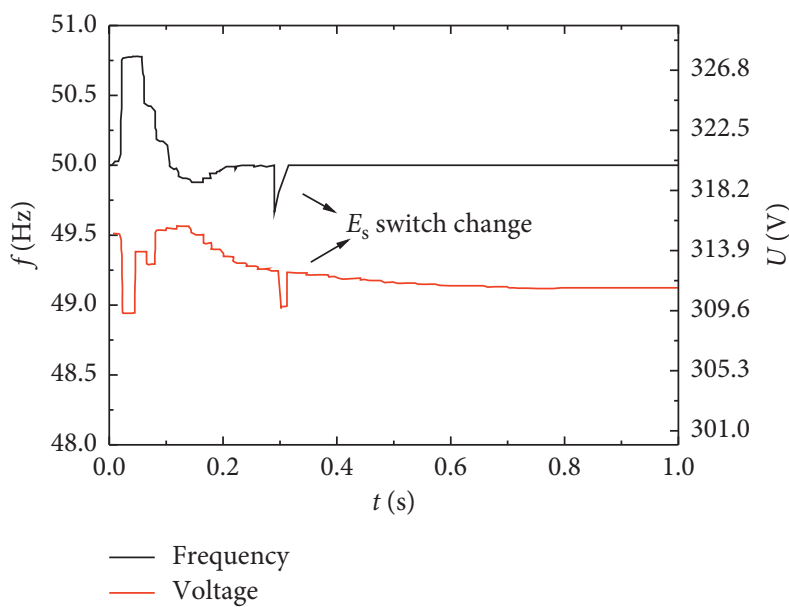

(b)

FigURe 8: Power balance dynamic adjustment. (a) Active and reactive dynamic adjustment. (b) Dynamic changes in voltage and frequency.

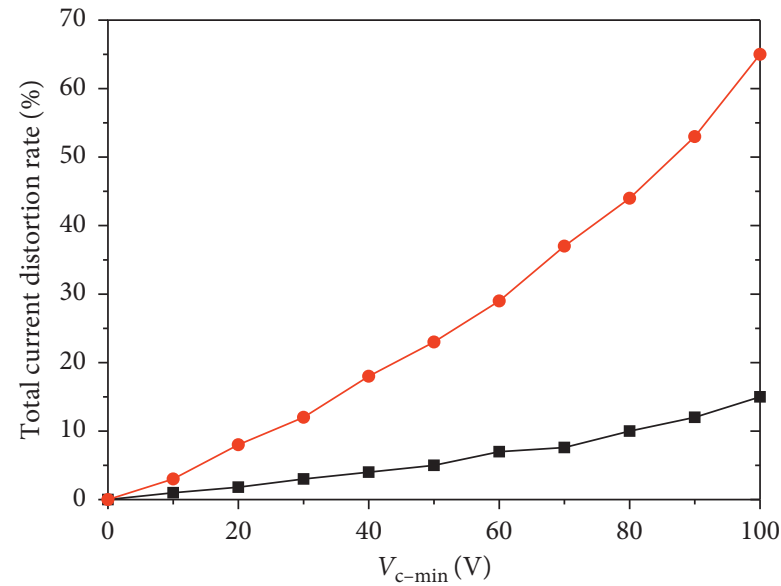

- Without third harmonic

$\rightarrow$ With third harmonic

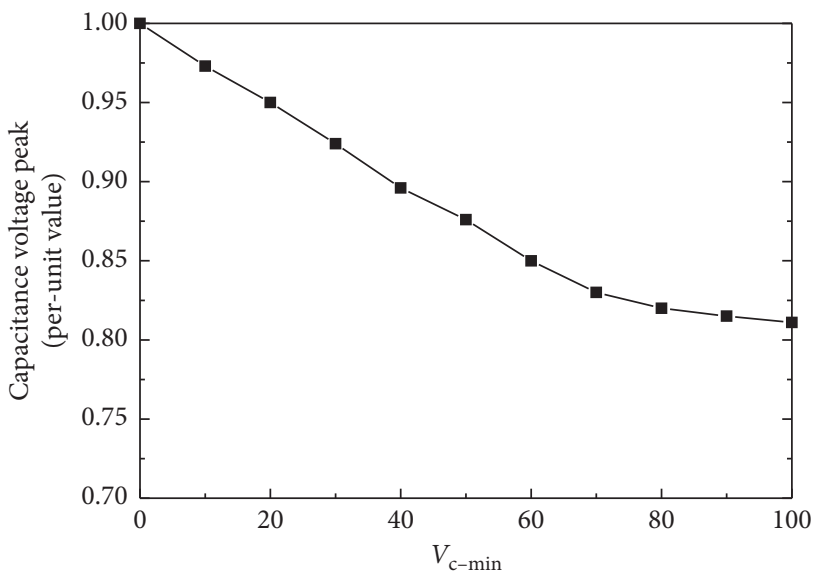

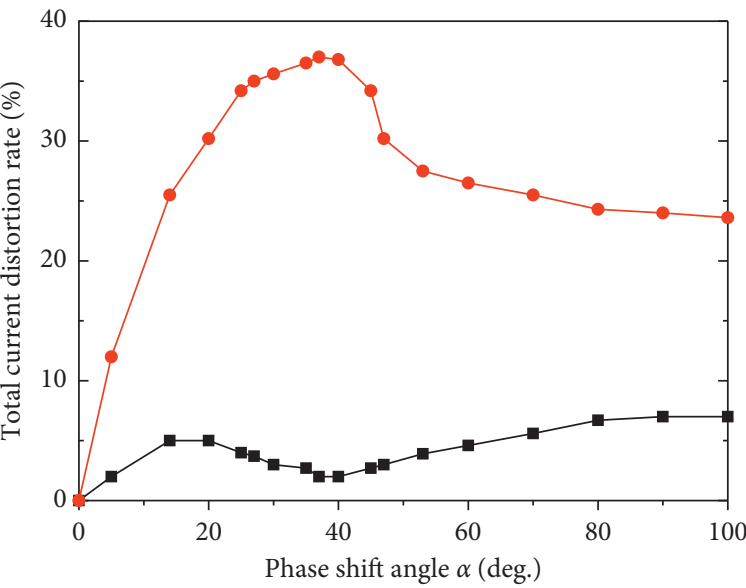

- Without third harmonic

$\rightarrow$ With third harmonic

(a)

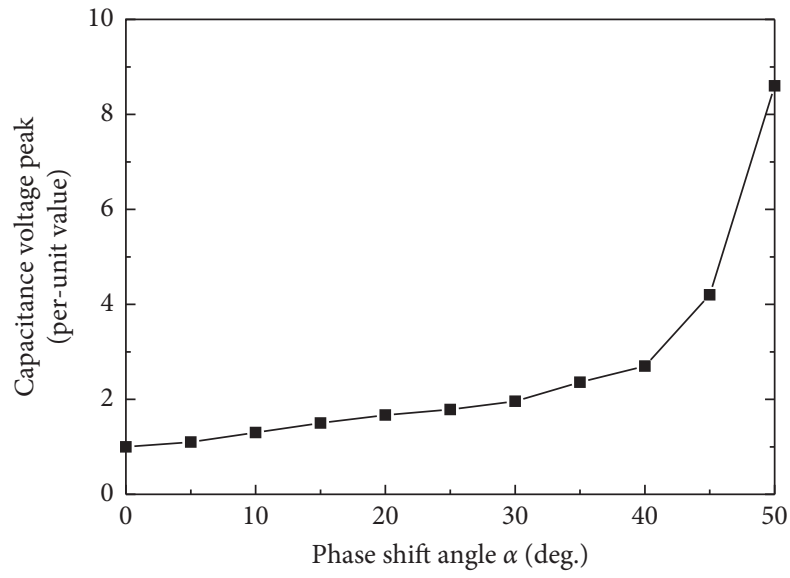

(b)

FIgURE 9: Reactive power parameter variation. (a) Current distortion rate change. (b) Change in capacitance voltage value. 


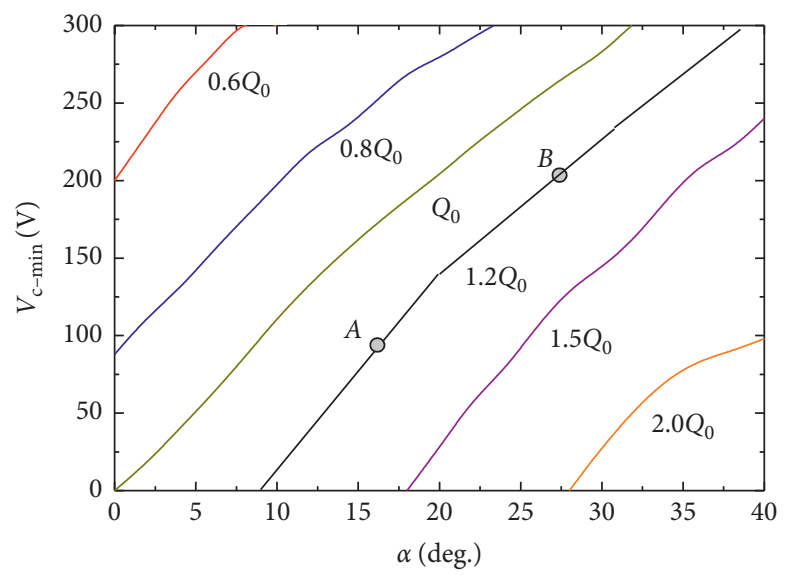

FIgURE 10: Equivalent reactive surface.
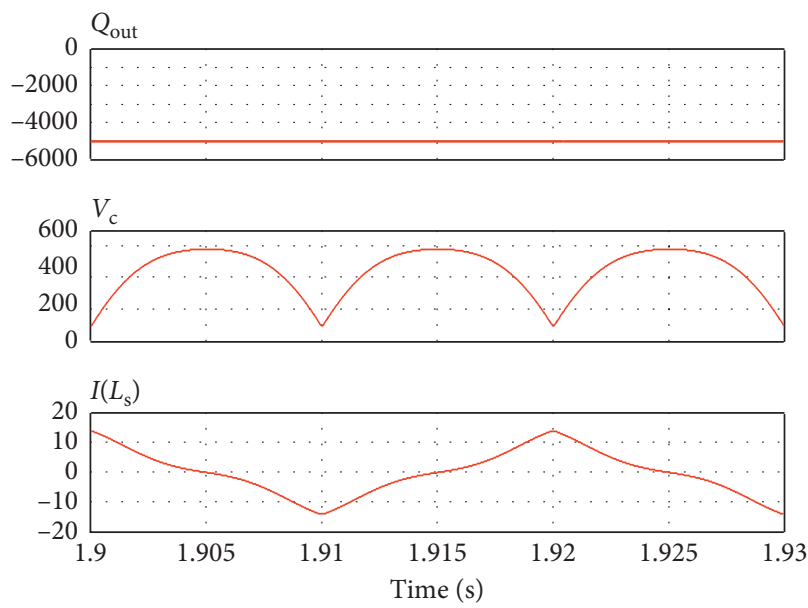

(a)
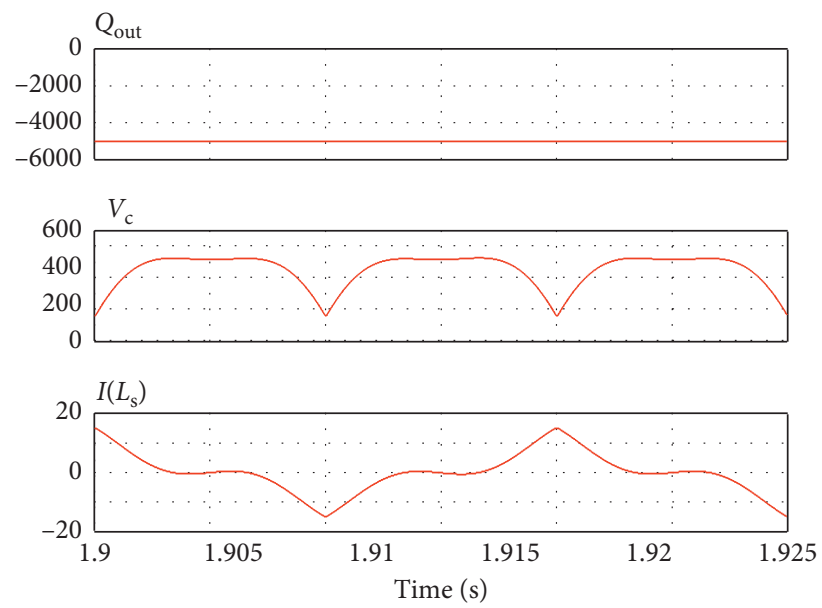

(b)

FIgURE 11: Comparison of reactive power optimization: (a) point $A$ before optimization; (b) point $B$ after optimization.

5.2. Experimental Verification. Through simulation, it can be seen that the energy storage structure of the full-bridge converter provides a flexible power regulation effect and it can realize a small capacitance and low harmonic operation. To verify the simulation, an experiment was conducted on a microgrid as shown in Figure 12.

The power generation unit includes an analog wind power generator and a photovoltaic array. The energy storage structure of the full-bridge converter includes a full-bridge converter, a battery, a DC-DC converter, and a variable load. The specifications are summarized in Table 2.

Figure 13 shows the experimental diagram of power regulation for an asynchronous microgrid. As shown in Figure 13, the stability of the microgrid is verified by increasing and decreasing the load. It can be seen that when the experimental system is stable, the voltage of the microgrid can be stabilized at $400 \mathrm{~V}$, and the root mean square of the grid voltage is stable when the experimental system is running stably. Further, the phase of voltage and current is corrected, and the power factor is increased to more than 95\%. However, some constraints must be considered, such as current distortion and the ability of the components to withstand voltage in practical application.

As shown in Figure 14, when the system is stable, the peak voltage and current distortion rate at point $A$ are measured. The yellow waveform stands for the voltage of phase A. The blue, purple, and green waveforms are the currents of the phase $A$, phase $B$, and phase $C$, respectively. At this point, the adjusting parameter is $(90,0 \mathrm{~V})$, the peak capacitance voltage can reach $580 \mathrm{~V}$, and the current distortion rate is $5.9 \%$. This is not the best working point. However, the optimal operation of the microgrid can be improved by dynamically adjusting $\alpha$ and $V_{\mathrm{c}-\mathrm{min}}$. After optimizing point $A(100,15 \mathrm{~V})$, the peak capacitance voltage is $490 \mathrm{~V}$ and the current distortion rate is $5.1 \%$. The experimental results show that the energy storage circuit structure of the full-bridge converter operates stably with a small capacitance and low harmonics with current distortion rate reduced by $0.8 \%$, realizes flexible power regulation, and restrains frequency and voltage fluctuations effectively at the same time. Thus, the power factor of the microgrid is 


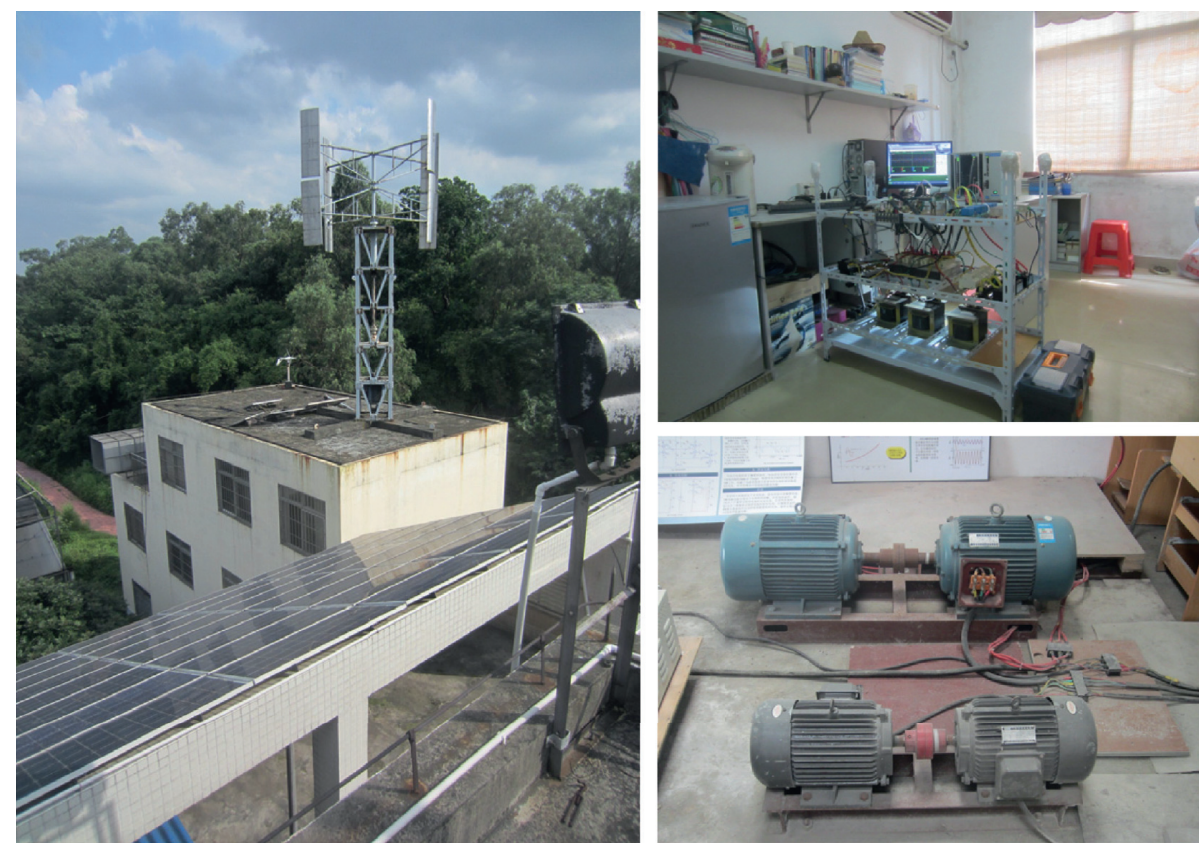

FIgURE 12: Experiment on microgrid system.

TABLE 2: Specifications of the experiment.

\begin{tabular}{|c|c|c|}
\hline Experiment platform & Element & Parameter \\
\hline \multirow{4}{*}{ Microgrid power generation system } & Rated power & $11 \mathrm{~kW}, 4 \mathrm{~kW}$ \\
\hline & Rated current & $23 \mathrm{~A}, 8.8 \mathrm{~A}$ \\
\hline & Rated voltage & $380 \mathrm{~V}$ \\
\hline & Frequency & $50 \mathrm{~Hz}$ \\
\hline \multirow{3}{*}{$\begin{array}{l}\text { Energy storage circuit structure of full-bridge } \\
\text { converter }\end{array}$} & DC capacitors & $40 \mu \mathrm{F}, 1250 \mathrm{~V}$ \\
\hline & 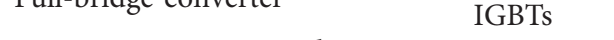 & $100 \mathrm{~A}, 1200 \mathrm{~V}$ \\
\hline & Storage battery & $200 \mathrm{~V}, 100 \mathrm{AH}$ \\
\hline \multirow{2}{*}{ DC-DC converter } & Energy storage inductor & $2 \mathrm{~mL}$ \\
\hline & $\begin{array}{r}\text { MOSFETs } \\
\end{array}$ & $10 \mathrm{kHZ}$ \\
\hline \multirow{3}{*}{ PV cell panel $\times 20$} & Maximum output power & $200 \mathrm{~W}$ \\
\hline & Open circuit voltage & $50.0 \mathrm{~V}$ \\
\hline & Short circuit current & $5.5 \mathrm{~A}$ \\
\hline Variable load & $\begin{array}{c}\text { Real power } \\
\text { Reactive power }\end{array}$ & $\begin{array}{c}0 \sim 15 \mathrm{~kW} \\
0 \sim 25 \mathrm{kVAr}\end{array}$ \\
\hline
\end{tabular}

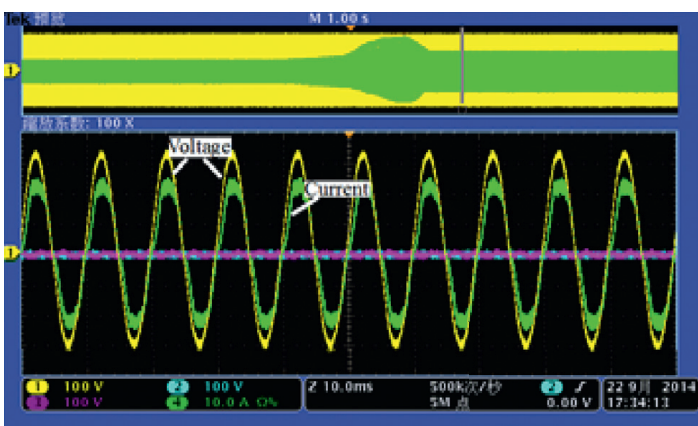

(a)

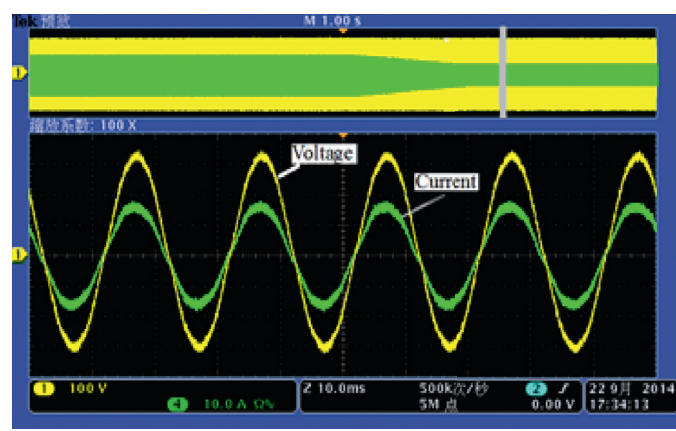

(b)

FIGURE 13: Power factor correction diagram of microgrid. (a) Load increases. (b) Load decreases. 

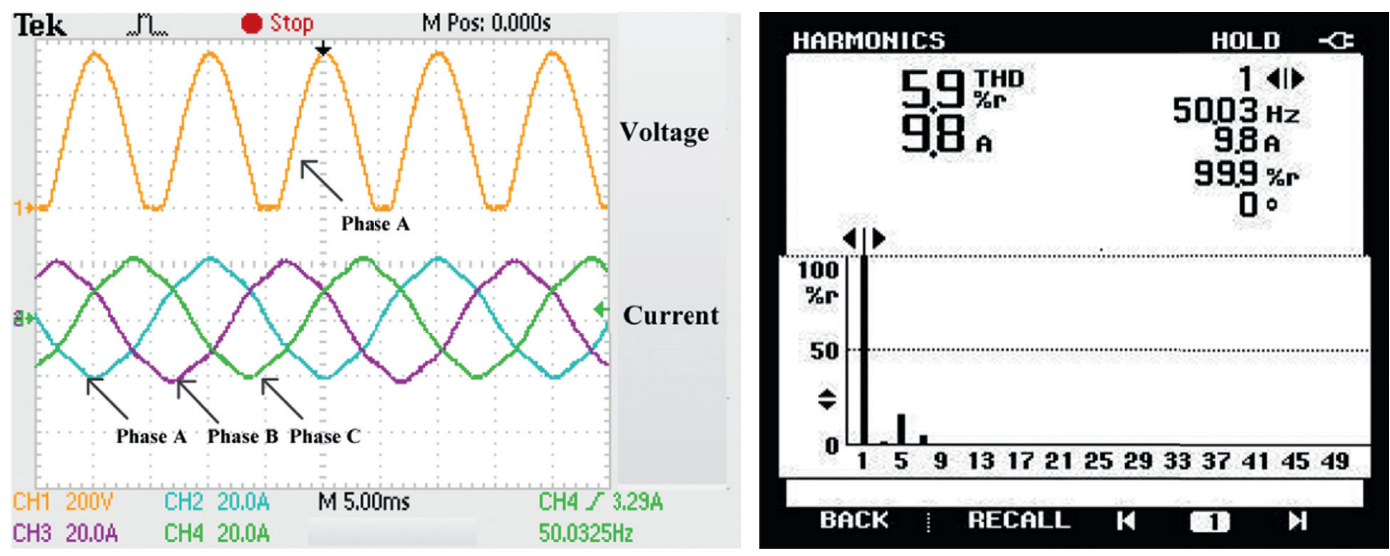

(a)
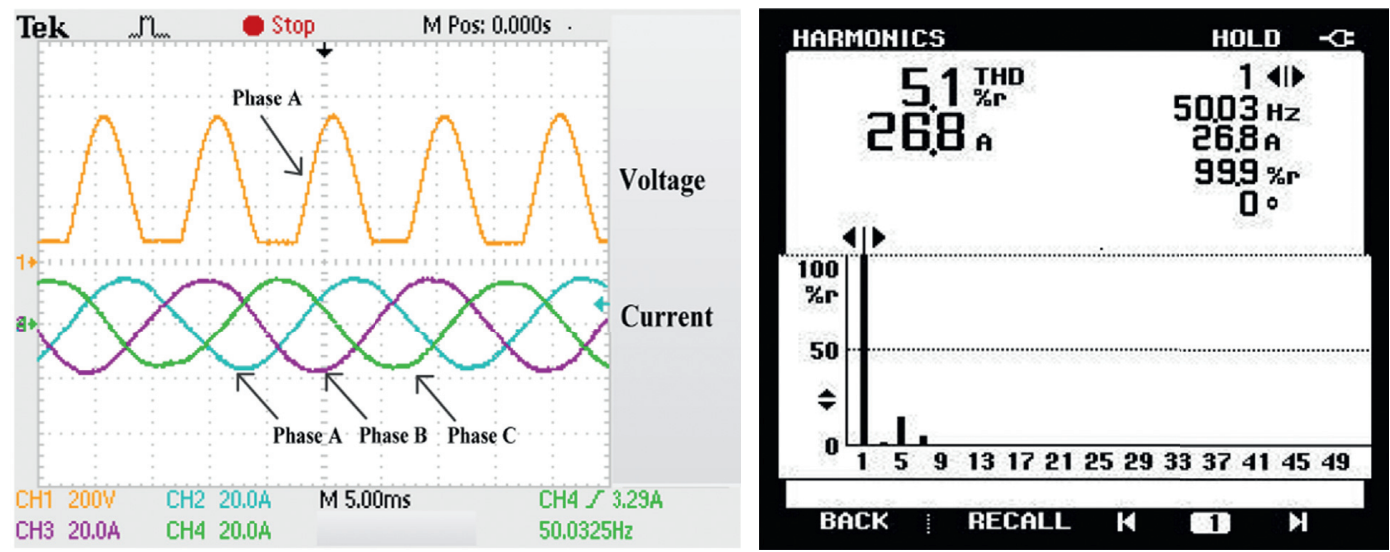

(b)

FIGURE 14: Optimal regulation of capacitance voltage peak and total harmonic distortion: (a) point $A$ before optimization; (b) point $A$ after optimization.

improved, thereby increasing the working efficiency of the electrical equipment and reducing the failure rate.

\section{Conclusion}

To address the power imbalance problem of microgrids, this paper proposed an energy storage circuit structure of a fullbridge converter from the perspective of inverter and capacitor charge/discharge, analyzed the working principle of the structure, and adopted a multimode power coordination strategy. The simulation proved that the structure can realize flexible power regulation, effectively suppress frequency and voltage fluctuations, and realize stable microgrid operation with small capacitance and low harmonics, which is of great significance for the promotion of microgrids.

\section{Data Availability}

The data used to support the findings of this study are available from the corresponding author upon request.

\section{Conflicts of Interest}

The authors declare that they have no conflicts of interest.

\section{Acknowledgments}

This research work was supported by the National Innovation and Entrepreneurship Training Program for College Students 201711075009.

\section{References}

[1] T. Kerdphol, F. S. Rahman, Y. Mitani, W. Masayuki, and H. Komsan, "Robust virtual inertia control to support frequency stability of an islanded microgrid," IET Renewable Power Generation, vol. 12, no. 7, 2018.

[2] B. Gultekin and M. Ermis, "Cascaded multilevel converterbased transmission STATCOM: system design methodology and development of a $12 \mathrm{kV} \pm 12 \mathrm{MV}$ ar power stage," IEEE Transactions on Power Electronics, vol. 28, no. 11, pp. 49304950, 2013.

[3] B. Singh, K. Al-Haddad, R. Saha, and A. Chandra, "Static synchronous compensators (STATCOM): a review," IET Power Electronics, vol. 2, no. 4, pp. 297-324, 2009.

[4] P. Gao, Study on the Planning of the Distributed Photovoltaic Power Access to the Middle and Low Rural Power Grid, North China Electric Power University, Beijing, China, 2017.

[5] W. Hu, J. Sun, Y. Jiang, and H. Zhou, "Low-frequency stabilization analysis of isolated micro-grid with STATCOM," Proceedings of the CSEE, vol. 35, no. 10, pp. 2454-2462, 2015. 
[6] T. Kawaguchi, T. Sakazaki, T. Isobe, and R. Shimada, "Offshore wind farm configuration using diode rectifier with MERS in current link topology," IEEE Transactions on Industrial Electronics, vol. 60, no. 7, pp. 2930-2937, 2013.

[7] Y.-L. Chen, H.-J. Chen, Y.-M. Chen, and K. H. Liu, “A stepping on-time adjustment method for interleaved multichannel PFC converters," IEEE Transactions on Power Electronics, vol. 30, no. 3, pp. 1170-1176, 2015.

[8] Y. U. Peng, L. L. I. U. Xinghua, S. Sun et al., "Study on operation control of island microgrid with high enewable energy penetration," Power System Technology, vol. 42, no. 3, pp. 779-788, 2018.

[9] Y. Liu and C. Wang, "Coordinated control strategy for microgrid with photovoltaic and energy storage units in grid connected operation," Proceedings of the CSU-EPSA, vol. 30, no. 1, pp. 127-132, 2018.

[10] L. Spasojevic, I. Papic, and B. Blazic, "Development of a control algorithm for a static VAR compensator used in industrial networks," Journal of Power Electronics, vol. 14, no. 4, pp. 754-763, 2014.

[11] B. Gultekin, C. O. Gercek, T. Atalik et al., "Design and implementation of a $154 \mathrm{kV}, 50$ MVAr transmission STATCOM based on 21-level cascaded multilevel converter," IEEE Transactions on Industry Applications, vol. 48, no. 3, pp. 1030-1045, 2012.

[12] Y. Wei, L. Kang, Z. Huang, Z. Li, and M. M. Cheng, "A magnetic energy recovery switch based terminal voltage regulator for the three-phase self-excited induction generators in renewable energy systems," Journal of Power Electronics, vol. 15, no. 5, pp. 1305-1317, 2015.

[13] R. K. Varma, S. A. Rahman, and T. Vanderheide, "New control of PV solar farm as STATCOM (PV-STATCOM) for increasing grid power transmission limits during night and day," IEEE Transactions on Power Delivery, vol. 30, no. 2, pp. 755-763, 2015.

[14] Q. Wang, H. E. Psillakis, and C. Sun, "Cooperative control of multiple agents with unknown high-frequency gain signs under unbalanced and switching topologies," IEEE Transactions on Automatic Control, vol. 64, no. 6, pp. 2495-2501, 2019.

[15] Z. Ding, "Adaptive consensus output regulation of a class of nonlinear systems with unknown high-frequency gain," Automatica, vol. 51, pp. 348-355, 2015.

[16] Q. Wang and C. Sun, "Adaptive consensus of multiagent systems with unknown high-frequency gain signs under directed graphs," IEEE Transactions on Systems, Man, and Cybernetics: Systems, pp. 1-6, 2018.

[17] R. Garmabdari, M. Moghimi, F. Yang, G. Evan, and L. Junwei, "Battery energy storage capacity optimisation for grid-connected microgrids with distributed generators," in Proceedings of the Universities Power Engineering Conference (AUPEC), IEEE, Melbourne, VIC, Australia, November 2017. 